\title{
Different significance of HRCT and FDG-PET/CT to predict lymph node status between patients with clinical stage IA lung adenocarcinoma and squamous cell carcinoma
}

\author{
Yasuhiro Tsutani ${ }^{1 *}$, Yoshihiro Miyata' ${ }^{1}$, Takeshi Mimura ${ }^{1}$, Masaoki Ito', Yuichiro Kai ${ }^{1}$, Atsushi Kagimoto', \\ Haruhiko Nakayama², Sakae Okumura ${ }^{3}$, Masahiro Yoshimura ${ }^{4}$, Morihito Okada ${ }^{1}$ \\ From World Society of Cardiothoracic Surgeons 25th Anniversary Congress, Edinburgh \\ Edinburgh, UK. 19-22 September 2015
}

\section{Background/Introduction}

True node-negative small sized non-small cell lung cancers are optimal candidates for sublobar resection without systematic lymph node dissection.

\section{Aims/Objectives}

The purpose of this study is to identify the predictive factors of true node-negative clinical stage IA non-small cell lung cancer.

\section{Method}

A multicenter database of patients with completely resected clinical stage IA lung adenocarcinoma $(n=502)$ or squamous cell carcinoma $(\mathrm{n}=100)$ was retrospectively analyzed. The relationship between lymph node status and preoperative factors such as tumor size on HRCT and maximum standardized uptake value (SUVmax) on FDG-PET/CT were examined.

\section{Results}

Multivariate analyses revealed that solid tumor size on HRCT (Odds ratio (OR), 1.42; $\mathrm{p}<0.001$ ) or SUVmax on FDG-PET/CT (OR, 1.04; $\mathrm{p}=0.049)$ was identified as an independent predictor of lymph node metastasis in patients with lung adenocarcinoma. The predictive criteria of node-negative lung adenocarcinoma were solid tumor size $<0.8 \mathrm{~cm}$ or SUVmax $<1.5$. Among patients who met the node-negative criteria, recurrence-free survival at 5 years was not significantly different between those who underwent lobectomy (96.0\%) and those who underwent sublobar resection (97.2\%). In patients with squamous cell carcinoma of the lung, no independent predictive factors for lymph node metastasis were identified in univariate or multivariate analysis.

\section{Discussion/Conclusion}

Either solid tumor size on HRCT or SUVmax on FDG-PET/CT was a significant independent predictor of nodal status in clinical stage IA lung adenocarcinoma. The node-negative criteria of solid tumor size $<0.8 \mathrm{~cm}$ or SUVmax $<1.5$ are helpful for choosing candidates for sublobar resection without systematic lymphadenectomy. In patients with clinical stage IA lung squamous cell carcinoma, systematic lymphadenectomy is advisable.

\section{Authors' details \\ 'Department of Surgical Oncology, Hiroshima University, Hiroshima, Japan. 2Department of Thoracic Surgery, Kanagawa Cancer Center, Yokohama, Japan. ${ }^{3}$ Department of Thoracic Surgery, Cancer Institute Hospital, Tokyo, Japan. ${ }^{4}$ Department of Thoracic Surgery, Hyogo Cancer Center, Akashi, Japan.}

Published: 16 December 2015

\section{doi:10.1186/1749-8090-10-S1-A332}

Cite this article as: Tsutani et al:: Different significance of HRCT and FDG-PET/CT to predict lymph node status between patients with clinical stage IA lung adenocarcinoma and squamous cell carcinoma. Journal of Cardiothoracic Surgery 2015 10(Suppl 1):A332. 